\title{
THE CLEARANCE OF BILIRUBIN FROM THE PLASMA. A MEASURE OF THE EXCRETING POWER OF THE LIVER
}

\author{
By A. A. WEECH, DOROTHEA VANN, ANd ROSE A. GRILLO \\ (From the Department of Pediatrics, College of Physicians and Surgeons, Columbia University, \\ and the Babies Hospital, New York)
}

(Received for publication January 20, 1941)

The clearance of bilirubin from the plasma following intravenous injection was first used to measure the excretory function of the liver by Eilbott (1) and Von Bergmann (2) in Germany in 1927. In the United States the method was introduced by Harrop and Barron (3) in 1931. Additional experiences by Soffer $(4,5,6,7,8)$ and by others $(9,10,11,12)$ have established the value of the test. Aside from the theoretical desirability of using a physiologic material (bilirubin) rather than a dye (bromsulphalein or rose bengal) to assess hepatic excretory function, it is generally agreed that the bilirubin test is unusually sensitive in demonstrating disturbed function when the degree of liver damage is relatively slight. The usefulness of the test and a desire to extend the range of clinical disorders to which it can be applied have prompted the present study.

As now used, the test involves determination of the percentage of injected bilirubin remaining in the circulation after a standard time period, usually 4 hours. Three samples of plasma are required. An initial sample $a$ is obtained to determine the natural level of bilirubin in the patient's plasma. Pure bilirubin-1 mgm. per kilogram of body weight dissolved in a small volume of $0.1 \mathrm{M}$ sodium carbonate solution-is injected intravenously. After allowing 4 to 5 minutes for mixing in the blood stream, a second sample of plasma $b$ is secured. The final sample $c$ is collected 4 hours later. If $B a, B b$ and $B_{c}$ represent the bilirubin concentrations in each of the three plasma samples, the percentage retention equals $100\left(B_{c}-B_{a}\right)$ $\div\left(B_{b}-B_{a}\right)$. Reported results indicate that in many normal subjects there is no retention of pigment at the end of the 4-hour period. Soffer (7) considers retention in excess of 5 per cent to be evidence of impaired liver function.

It will be observed that the above method which utilizes percentage retention as the measure of hepatic function is based on the assumption that rate of excretion is not influenced by the basal level of bilirubin in the plasma. The value for this level is simply subtracted from each of the other levels before the retention is computed. It will appear presently that the assumption is not justified. However, since most authors state that the test should not be applied when the basal level is above $1 \mathrm{mgm}$. per cent, and since the result is usually interpreted simply as normal or abnormal, and not as a quantitative measure of excretory function, it has in general served its purpose satisfactorily. Occasional attempts $\left(13,14^{1}\right)$ to utilize the test when the basal concentration was elevated have yielded results which are difficult to interpret.

In extending the range of disorders to which the bilirubin excretion test can be applied, it is necessary to recognize that probably no test of excretory function can yield meaningful results when the plasma van den Bergh reaction is direct. The direct reaction is generally assumed to mean that bile is regurgitating from the biliary tract into the blood; if the assumption is true, a part of the material excreted by the liver is returned to the blood and estimations of blood level cannot be expected to measure excretion alone. In hemolytic disease the reaction is indirect, there is no evidence for regurgitation, and it should be possible to assess excretory function even though the basal concentration is high. The theory to be described provides a rational basis for interpreting the test in this type of case; the estimate of liver function which it yields is quantitative and independent of the basal concentration of bilirubin in the plasma.

\section{THEORY}

Three assumptions are required in developing the theory of bilirubin clearance. Two of these

1 These investigators studied the liver function of a patient with chronic hyperbilirubinemia, moderate splenomegaly and hepatomegaly, and march hemoglobinuria. The conventional bilirubin excretion test showed no retention of injected pigment after 3 hours, that is, plasma bilirubin concentration had returned to its previous equilibrium level of $3.0 \mathrm{mgm}$. per cent. Later, a bilirubin clearance test performed by the method of this paper disclosed marked impairment of hepatic excretory function. 
are entirely reasonable; the third is an approximation. The first assumption states that the level of bilirubin normally circulating in the plasma is the result of an equilibrium between the rate of formation and the rate of excretion. Accordingly, in the equations which follow the basal bilirubin concentration will be represented by the symbol $L_{\text {eq. }}$ which is to be read "level at equilibrium." The rates of formation and excretion will be expressed by the letters $F$ and $E$, respectively. The second assumption is that the rate of bilirubin formation will not change significantly during the several hours when a test is being run. $F$ is therefore a mathematical constant. The third assumption states that the velocity of bilirubin excretion is at any moment proportional to the square of its concentration in the plasma. If the changing concentrations after an intravenous injection are represented by the symbol $L$ and the constant of proportionality by $K$, it follows that instantaneous excretion equals $K L^{2}$. These assumptions will now be used for a mathematical statement of the theory; at a later place their validity will be examined more closely.

It is axiomatic to say that any change in the amount of bilirubin in each $100 \mathrm{cc}$. of plasma during a given time period will equal the difference between bilirubin added to and bilirubin taken away from the $100 \mathrm{cc}$. of plasma. Briefly, change $=$ formation - excretion. Since the equality holds no matter how brief the time interval, the change in the plasma level can be replaced by its derivative with respect to time; that is,

$$
\text { 1. } \frac{d L}{d t}=F-E \text {. }
$$

It has been assumed that the instantaneous velocity of excretion is equal to $K L^{2}$; accordingly, the equation takes the form:

2.

$$
\frac{d L}{d t}=F-K L^{2} .
$$

The constant $K$ in this equation can be evaluated to express for each 100 cc. of plasma the rate of excretion of bilirubin in milligrams per minute per milligramsquared of concentration in the plasma. Accordingly, "the velocity constant" is a measure of the excretory power of the liver. The higher the value of $K$, the greater is the ability of the liver to excrete bilirubin.

Equation 2 can be used to derive the relationship which describes the path of bilirubin concentration in the plasma following an intravenous injection. The derivation is essential to test the reliability of the theory and to compute the velocity constant. We first observe that the rate of change in concentration, $d L / d t$, is zero when equilibrium has been reached between formation and ex- cretion. It follows from equation 2 that $F=K L$ eq. ${ }^{2}$. Substitution in equation 2 yields

3. $\frac{d L}{d t}=K L_{\mathrm{eq} .}{ }^{2}-K L^{2}=-K\left(L^{2}-L_{\mathrm{eq} .}{ }^{2}\right)$.

Transposition of terms leads to

4.

$$
\frac{d L}{L^{2}-L_{\text {eq. }}{ }^{2}}=-K d t \text {. }
$$

Equation 4 is in a form which can be integrated. When this step has been performed and the constant of integration evaluated by introducing a value $L_{0}$ which represents the bilirubin concentration when time is zero, that is, the first measurement after the injection, the following relationship emerges:

5. $\log \left(\frac{L+L_{\text {eq. }}}{L-L_{\text {eq. }}}\right)=\log \left(\frac{L_{0}+L_{\text {eq. }}}{L_{0}-L_{\text {eq. }}}\right)+\frac{K L_{\text {eq. }}}{1.1513} t$.

Equation 5 expresses the variations in plasma bilirubin level $L$ with respect to time $t$ which is conveniently measured in minutes after the first post-injection sample is withdrawn. The value 1.1513 is a constant which enters in the conversion of natural to ordinary logarithms. It will be observed that values for the lefthand term of the equation can be computed readily from analyses at different time intervals and that these values are linear with respect to time. When multiple serial measurements have been made, the procedure for obtaining the velocity constant is to prepare a graph on which these values are plotted as ordinates against time as abscissas. A straight line is then drawn as closely as possible through the consecutive points. The slope $m$ of the line will then correspond to the coefficient of $t$; that is,

and

$$
m=\frac{K L_{\mathrm{eq}}}{1.1513}
$$

6.

$$
K=\frac{1.1513 \times m}{L_{\mathrm{eq}}} .
$$

When $K$ has been determined, equation 5 can be used to calculate theoretical values of $L$ for a number of values of $t$. Plotting these data with $L$ as ordinate and $t$ as abscissa yields the theoretical curve of clearance; direct comparison between theoretical and observed concentrations is then possible. Equation 5, as given, presupposes that one of the observational points, namely $L_{0}$, will lie exactly on the theoretical curve. Often a better fit to all of the data can be obtained if this limitation is not imposed. If the equation for the straight line yielded by the logarithmic plot is written in the form $y=m t$ $+b$, it is clear that values of $\boldsymbol{y}$ computed for different values of $t$ will correspond to the left-hand term of equation 5 for the same values of $t$. The theoretical concentrations $L$ can then be obtained. If $\alpha$ equals the number whose logarithm is $y$, then

and

$$
\alpha=\frac{L+L_{\text {eq. }}}{L-L_{\text {eq. }}}
$$

7.

$$
L=\left(\frac{\alpha+1}{\alpha-1}\right) L_{\mathrm{eq} .} .
$$


The reader should note that the equations of this section are concerned with the development of a theory and with putting the data in such form that the agreement between theory and observation can be tested. Later it will be shown that the velocity constant, which is the measure of hepatic function, can be calculated in a simple way from observational data without recourse to graphic methods.

\section{Agreement between observation and theory}

The theory outlined above intimates that the quantitative appraisal of hepatic excretory function given by the velocity constant is independent both of the amount of bilirubin injected and of the exact time intervals at which samples are withdrawn. However, practical considerations suggest that sufficient bilirubin be injected and samples be taken over long enough time intervals so that the consecutive concentrations cover a range well outside the limits of analytical error. In the observations to be described, the amount of bilirubin injected was $5 \mathrm{mgm}$. per kilo. An injection of this size has never given rise to toxic manifestations.

According to the size of the patient (children), the bilirubin was dissolved in from 5 to $10 \mathrm{cc}$. of a $0.1 \mathrm{M}$ solution of sodium carbonate. Solution was hastened by warming over a flame; after cooling to body temperature it was injected without further sterilization. A blood sample was taken before injection to determine the value of Leq. ; after injection 5 to 7 minutes were allowed for mixing in the circulation; then, a number of samples were obtained over a period of 4 hours. Analyses were made on serum rather than plasma to eliminate the osmotic action of the ordinary anticoagulants.

Data obtained with A. S., a Jewish boy, aged 12 years, are presented in graphic form in Figure 1. This patient entered the hospital with splenomegaly resulting from fibrosis and obstruction of the larger branches of the splenic vein. The case is selected for illustration because at operation (splenectomy) a biopsy specimen was secured from the liver. The liver tissue was normal. The bilirubin clearance test was carried out in the manner outlined above. The upper chart in Figure 1 is a plot of the logarithms of $\left(L+L_{\text {eq. }}\right)$ $\div\left(L-L_{\text {eq. }}\right)$ against the time at which the samples were taken. The straight line through the plotted points was fitted by eye and its equation written in the form shown. By equation 6 the
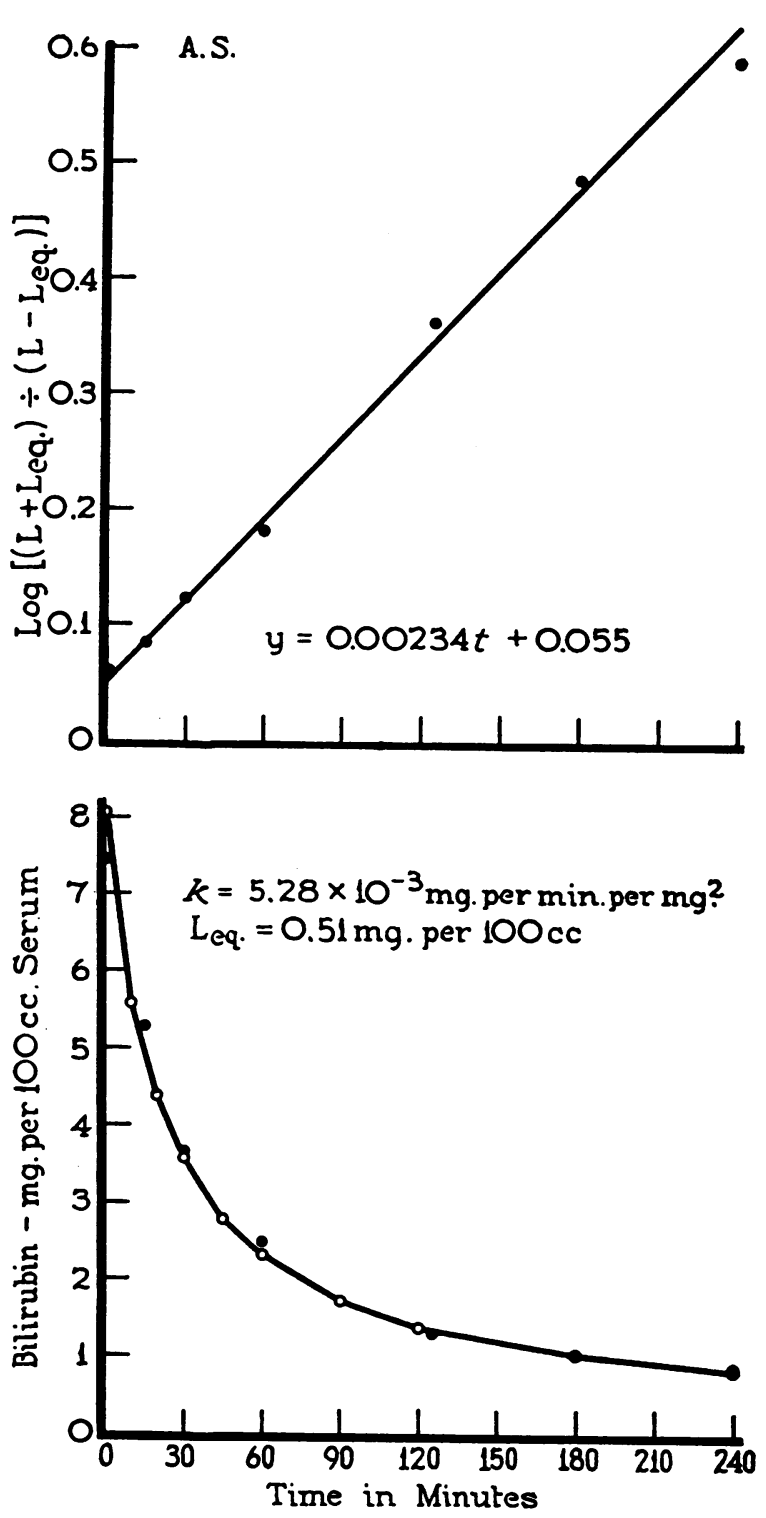

Fig. 1. Bilirubin Clearance in a Boy with Normal Liver Function

The upper chart illustrates the graphic method of fitting a straight line to the observations. In the lower chart the line joining the open circles depicts the theoretical curve of clearance; the black dots refer to analytical data and show the agreement between observation and theory.

velocity constant of excretion was calculated to be $5.28 \times 10^{-3} \mathrm{mgm}$. units. ${ }^{2}$ Then values of $y$

2 For the sake of brevity the expression " milligram units" will be used throughout the rest of this paper rather than the more exact "milligrams per minute per milligram-squared of concentration in the plasma." 
were computed for a number of values of $t$; by equation 7 the theoretical bilirubin concentrations corresponding to the time intervals were determined. The lower curve of Figure 1, plotted from these data, shows the theoretical curve of clearance; the black dots along the general path of the curve depict the observed concentrations. It is clear in this case that the theory describes the findings with close approximation. Inspection of the logarithmic graph in Figure 1 shows that the values of the ordinate for low values of $t$ have been fitted closely to the straight line, whereas with higher values of $t$ the fit is less close; nevertheless the clearance curve lies near to all of the data. This circumstance results from the nature of the function and indicates a general principle to be followed in obtaining the theoretical curve; the logarithmic values corresponding to high concentrations and low time intervals must be fitted closely by the line, whereas with the later values from lower concentrations considerable latitude is permissible.

In Figure 2 are presented data obtained with L. H., a white girl, aged 9 years. An enlarged, firm liver, an abnormal galactose tolerance test, and a history of an acute illness $2 \frac{1}{2}$ years earlier with jaundice which persisted several months suggested a diagnosis of cirrhosis. The findings in the bilirubin clearance test illustrate the application of the theory to a case in which liver function was impaired without elevation of the equilibrium level. The agreement between observation and theory is adequate, though less close than in most other cases.

Figure 3 presents data given by R. R., aged 6 years, a boy of Italian parentage suffering from chronic erythroblastic anemia. In this case the basal bilirubin concentration in the serum was elevated to $2.45 \mathrm{mgm}$. per $100 \mathrm{cc}$. and there was chronic jaundice. The agreement between observation and theory is good. The velocity constant, $1.23 \times 10^{-3} \mathrm{mgm}$. units indicates marked impairment in the excretory function of the liver.

\section{Validity of the basic assumptions}

The illustrative cases of the preceding section show that the theory of bilirubin excretion is able to chart the path of clearance from the plasma with approximate accuracy. The illustrations are
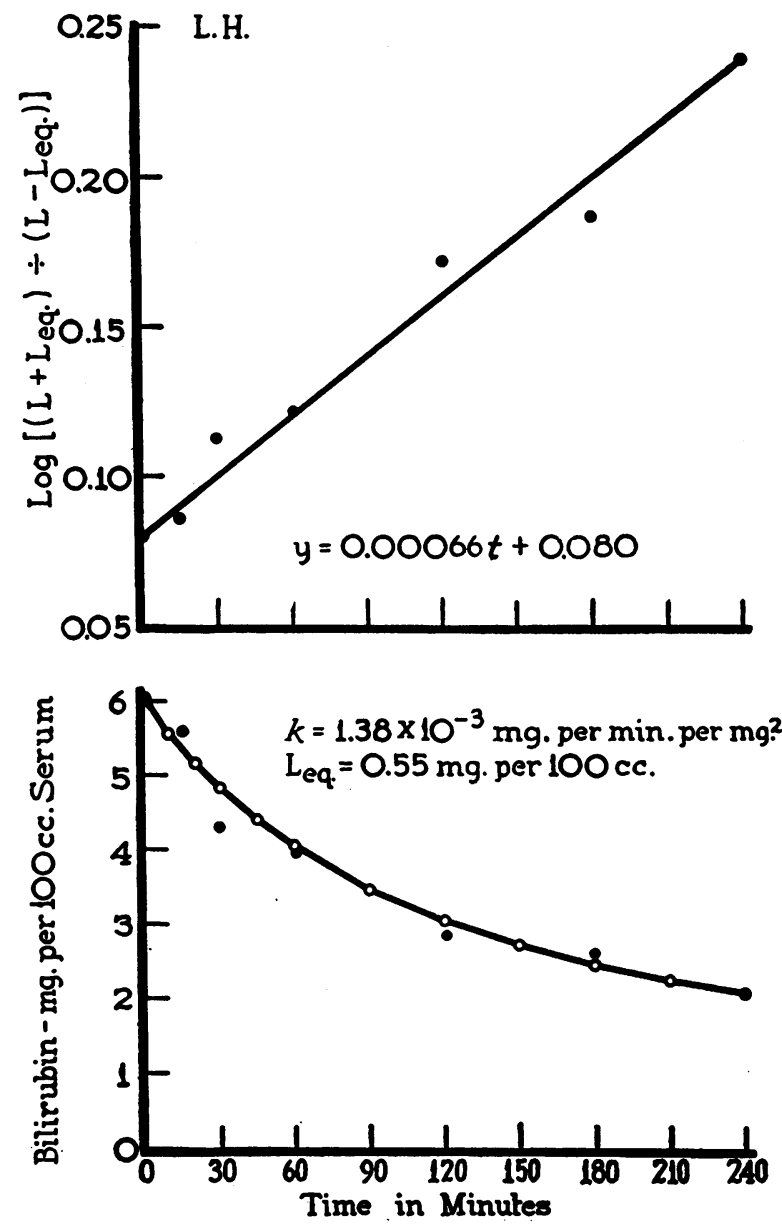

Fig. 2. Bilirubin Clearance in a Girl with Impaired Liver Function in the Presence of a Normal Basal Concentration of Bilirubin in the Plasma

The upper and lower charts have the same meaning as in the previous figure.

average ones; in a number of instances the goodness of fit between observation and theory has been closer than in any of the cases presented. It does not necessarily follow that the assumptions underlying the theory are justified; if they are not, the quantitative estimate of liver function can be unreliable. It is therefore important to examine the assumptions in greater detail.

The first assumption was that the normal level of bilirubin in the plasma results from an equilibrium between the rates of formation and excretion. The alternate possibility is that the liver establishes a threshold level above which bilirubin is excreted and below which it is not. The wide range of normal levels encountered in different 
patients, and the fact that high levels in hemolytic disease are invariably associated with demonstrably slow excretion of injected bilirubin, speak against a threshold mechanism as being the only factor in maintaining equilibrium values. Moreover, it is
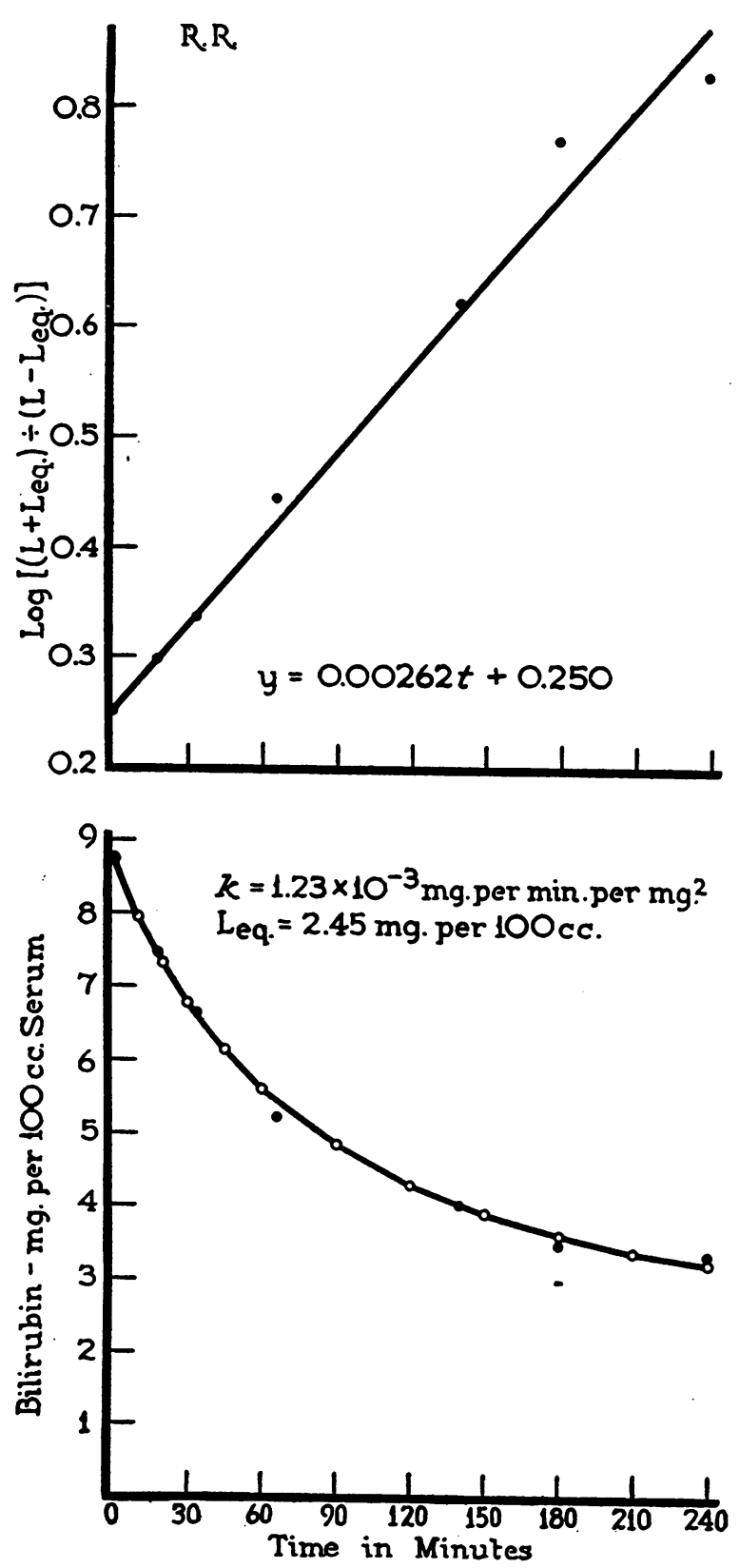

Fig. 3. Bilirubin Clearance in a Boy with Chronic Erythroblastic Anemia

The findings illustrate application of the theory when liver function is impaired and when the basal serum bilirubin concentration is elevated. possible to show that an increase in the rate at which bilirubin is added to the circulation will result in a new and elevated level of equilibrium. An experiment of this type performed on a dog which weighed 20 kilos is illustrated in Figure 4.

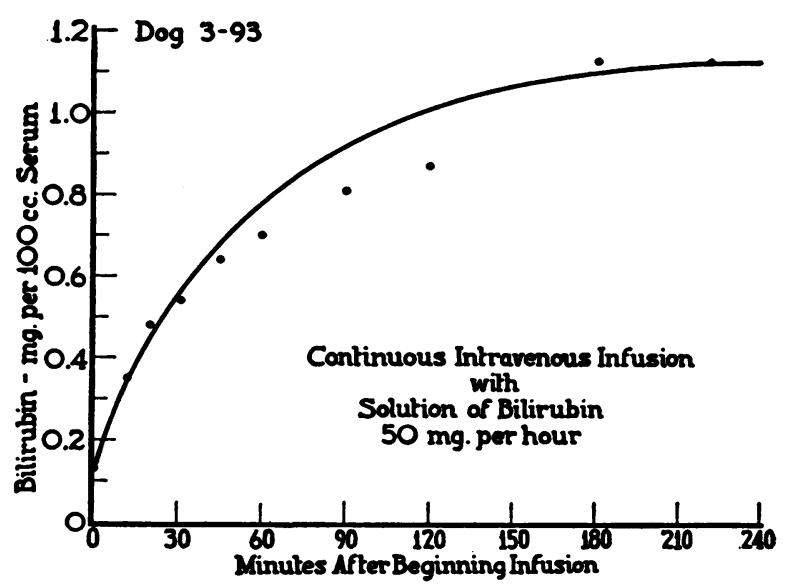

Fig. 4. An Experiment with a Dog Which Demonstrates that the Continuous Intravenous Infusion of Bilirubin Leads to a New EQuilibrium Concentration in the Serum at an Elevated Level

At the start the bilirubin concentration in the plasma was $0.13 \mathrm{mgm}$. per $100 \mathrm{cc}$. During the next 4 hours the dog received a continuous intravenous infusion of bilirubin solution; by means of special apparatus the rate of flow was maintained constant at $13 \mathrm{cc}$., containing $50 \mathrm{mgm}$. bilirubin, per hour. Serial determinations showed an initially rapid, then progressively slower, rise in serum bilirubin concentration until a new equilibrium was reached at $1.13 \mathrm{mgm}$. per $100 \mathrm{cc}$. Experiments of this kind provide additional grounds for believing that the equilibrium level is affected by the rate of bilirubin formation. They do not, however, exclude the possibility of a basal threshold level which would be reached, but not passed, if all formation stopped. On anatomical grounds it appears unlikely that the liver can function as an excretory threshold organ and we know of no other evidence to indicate that it can. Proof that it cannot is, however, lacking and the first proposition underlying the theory must remain a reasonable assumption.

The second assumption that the rate of bilirubin formation will remain constant during the period of the test rests on the observation that individual subjects tend to maintain the same 
plasma concentrations when studied serially over a number of days and on the fact that high concentrations such as are encountered in hemolytic disease do not affect the stability of the equilibrium level. It is reasonable, then, to suppose that even greater constancy will exist during the limited 4-hour period of a test. Presumably, the rate of bilirubin formation is related closely to the rate of blood destruction; the latter appears to proceed at a uniform rate both in normal subjects and in patients with hemolytic disease. In occasional conditions, as in the early neonatal period, the rate of blood destruction may be changing rapidly; under such circumstances, the theory developed in this paper would have to be applied with great caution.

The third asumption that the rate of excretion is proportional to the square of the concentration in the plasma is admittedly an approximation. Inspection of the graphs presented in this paper, as well as of similar data charted by Eilbott (1) and by Harrop and Barron (3), leaves no doubt of a direct relationship between concentration and rate of excretion; after intravenous injection the rate of disappearance of bilirubin from the plasma always decreases as the concentration declines. Unless velocity of excretion increased with rising concentration, no equilibrium could be reached in experiments of the type illustrated in Figure 4. It is clear, then, that $E$, the excretory rate, can be represented by some mathematical function of $L$, the plasma concentration. There is no proof, however, that the function will be the same for all subjects; at the outset it is possible that the nature of the function may be altered in the presence of liver disease. The function represented by the equation, $E=K L^{2}$, was arrived at after experimenting with a number of other relationships. In particular it was found that the simpler expression, $E=K L$, is not adequate to describe observational findings. On the contrary, the expression, $E=K L^{2}$, has led to theoretical clearance curves which agree satisfactorily with observation in a wide variety of patients and in dogs. Cumulative experience, however, stresses the approximate nature of the relationship. Examination of the average findings in a series of patients indicates that somewhat better agreement with theory would result if, in the general ex- pression, $E=K L^{n}, n$ were assigned a value somewhat greater than 2 . The difficulty of handling such expressions mathematically has prevented further exploration along this line. Because the relationship used in the theory is only a good approximation, it follows that the measure of liver function $K$ will not, for any one patient, be entirely independent of the amount of bilirubin injected nor of the time interval over which samples are collected. With intervals ranging from 2 to 4 hours, the variations in $K$ are small but it is probably inadvisable to rely on velocity constants computed from data covering a shorter period than 2 hours. We do not have experience with smaller injections than $5 \mathrm{mgm}$. per kilo.

\section{Direct computation of the constant of bilirubin excretion}

The illustrative data which have been presented so far and the method of computing the velocity constant, have been given in order to establish the validity of a theory. Actually, the assessment of hepatic excretory function can be accomplished when only three samples of serum are analyzed.

The level in the circulation before injecting bilirubin, $L_{\text {eq. }}$ must be measured first. When sufficient time has elapsed after the injection for thorough mixing, a second sample, $L_{1}$, is obtained. Finally, after 2 to 4 hours, the third sample, $L_{2}$, is secured. If $t_{1}$ and $t_{2}$ represent the time in minutes corresponding to the samples $L_{1}$ and $L_{2}$, which express concentration in milligrams per $100 \mathrm{cc}$. serum, the value of the velocity constant $K$ can be computed from the equation:

$$
\begin{gathered}
\text { 8. } \quad K=\frac{1.1513}{L_{\text {eq. }\left(t_{2}-t_{1}\right)}} \log \frac{\left(L_{2}+L_{\text {eq. }}\right)\left(L_{1}-L_{\text {eq. }}\right)}{\left(L_{2}-L_{\text {eq. }}\right)\left(L_{1}+L_{\text {eq. }}\right)} \text {. } \\
\text { Error of measurement }
\end{gathered}
$$

In the bilirubin tests carried out in the Babies Hospital a fairly large number of samples has been analyzed with each patient in order to chart the path of the clearance curve. By use of equation 8 such data permit several independent calculations of the velocity constant. Since an approximation is involved in the theory, the agreement to be expected among the several values will depend to some extent on the time periods used in their computation. We have already stated the desirability of a time interval of at least 2 hours. In most of our cases three samples, $L_{1}, L_{2}$ and $L_{3}$, were obtained during the first 30 minutes after injection of bilirubin; subsequent samples $L_{4}, L_{3}$ 
and $L_{6}$, were taken at 120 minutes, at 180 minutes and at 240 minutes, respectively. Illustrative values of $K$ computed from $L_{1}$ and $L_{4}, L_{2}$ and $L_{3}$, $L_{3}$ and $L_{6}$, are recorded in Table I. If the func-

TABLE I

Agreement among three independently calculated values of the velocity constant

The figures in column A were computed from samples taken at 0 and 120 minutes, in $B$ from samples at 15 and 180 minutes, in $C$ from samples at 30 and 240 minutes. The illustrative cases were selected to sample the range of average values which have been encountered.

\begin{tabular}{|c|c|c|c|c|}
\hline \multirow{2}{*}{ Patient } & \multicolumn{4}{|c|}{$K \times 10^{-3} \mathrm{mgm}$. per minute per mgm. ${ }^{2}$} \\
\hline & $\mathbf{A}$ & B & C & Average \\
\hline 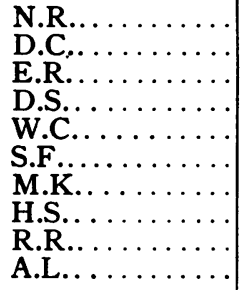 & $\begin{array}{r}26.2 \\
9.1 \\
7.5 \\
5.9 \\
4.9 \\
4.4 \\
3.8 \\
2.3 \\
1.2 \\
1.3\end{array}$ & $\begin{array}{r}26.9 \\
9.9 \\
8.0 \\
6.4 \\
6.2 \\
5.2 \\
3.5 \\
2.4 \\
1.4 \\
1.1\end{array}$ & $\begin{array}{r}20.4 \\
8.5 \\
7.4 \\
6.1 \\
4.8 \\
4.5 \\
2.8 \\
2.3 \\
1.1 \\
0.9\end{array}$ & $\begin{array}{r}24.5 \\
9.2 \\
7.6 \\
6.1 \\
5.3 \\
4.7 \\
3.4 \\
2.4 \\
1.2 \\
1.1\end{array}$ \\
\hline
\end{tabular}

tion of the liver is to be assessed from the minimum number of analyses, and if the two samples which follow the injection are separated by an interval of at least 2 hours, data like those in Table I can be used to determine the error of the assessment. Examination of the separate $K$ values yielded by the 35 patients studied so far shows that, although the error becomes larger as the value of $K$ rises, the percentage error is approximately constant for all values of $K$. This error (coefficient of variation) is \pm 13.6 per cent. When several independently determined values are averaged, the error will be reduced. Since the range of $K$ encountered in different subjects, both diseased and healthy, has been from $1.09 \times 10^{-3}$ to $24.5 \times 10^{-8} \mathrm{mgm}$. units, the 13.6 per cent error will not in most instances interfere with adequately accurate appraisal of liver function. Only in those cases where function is within or near the zone which separates normal from abnormal is greater accuracy desirable and in such cases a larger number of samples should be taken.

\section{Clinical findings}

The measures of liver function, that is, $K$ values, to be reported were determined by the method of averaging several independent estimates. In most instances three, in a few only two, such estimates were utilized. Experience shows that these average values yield theoretical clearance curves which agree with observation as well as, and often better than, the values obtained by the graphic method. The agreement between values determined by the two methods has always been close.

The 35 patients whose excretory liver function has been measured were selected from the wards of the Babies Hospital. They ranged in age from 8 months to 15 years. Since within these limits there is no evidence that function varies with age, this factor will not be considered further. No single group of the patients can be set aside as representing normal healthy controls since such subjects do not constitute the population of a hospital. In the majority of instances, however, there was no reason to suspect the presence of impaired liver function; a few patients were studied because of chronic hemolytic jaundice, a few because of known cirrhosis of the liver, and in a few the status of the liver was in doubt. None of the patients had fever when the tests were done.

With 17 of the 35 patients the velocity constant was above $5.0 \times 10^{-3} \mathrm{mgm}$. units; in none of these cases was there reason to suspect impaired liver function and the $K$ values are clearly within the range of normal. The highest excretory rate in the group, $24.5 \times 10^{-3} \mathrm{mgm}$. units, was recorded in a colored boy, aged 11 years, who presented a cardiac murmur and a story of recurrent arthralgia over a number of years. There was no past episode of cardiac decompensation and the possible diagnosis of rheumatic fever was never clearly established.

Findings with the 18 patients who exhibited constants below $5.0 \times 10^{-3} \mathrm{mgm}$. units are recorded in Table II. Inspection of the diagnoses for the first 6 patients, with constants ranging from 4.88 to $3.63 \times 10^{-3} \mathrm{mgm}$. units, suggests that these values must likewise be regarded as normal. The seventh patient, R. H., yielded a constant of $3.36 \times 10^{-3}$ units; he had suffered for some years with cirrhosis of the liver, proven by biopsy. There was chronic ascites for which abdominal paracentesis had been performed on numerous occasions. However, the history failed to reveal any episode of jaundice, the basal level of bilirubin in his plasma was relatively low, and it seems likely that hepatic excretory function was 
TABLE II

Findings with 18 patients in whom the velocity constant of bilirubin clearance, $K$, was less than $5.0 \times 10^{-3} \mathrm{mgm}$. per minute per milligram-squared of concentration in the plasma

\begin{tabular}{|c|c|c|c|c|}
\hline Patient & Age & $L_{\text {eq. }}$. & $K$ & Diagnosis \\
\hline 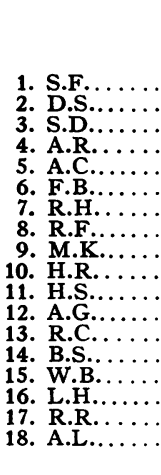 & $\begin{array}{r}\text { years } \\
2 \\
2 \\
1 \\
1 \\
4 \\
6 \\
7 \\
11 \\
11 \\
7 \\
9 \\
12 \\
2 / 3 \\
2 \\
9 \\
13 \\
9 \\
6 \\
15\end{array}$ & $\begin{array}{c}\text { mgm. } \\
\text { per } \\
100 \text { cc. } \\
0.86 \\
0.50 \\
0.72 \\
0.73 \\
0.52 \\
0.73 \\
0.36 \\
0.99 \\
0.59 \\
0.92 \\
0.76 \\
1.80 \\
0.69 \\
0.86 \\
1.84 \\
0.55 \\
2.45 \\
1.40\end{array}$ & $\begin{array}{c}\text { mgm. } \\
\text { units } \\
\times 10^{-3} \\
4.88 \\
4.69 \\
4.54 \\
4.50 \\
3.90 \\
3.63 \\
3.36 \\
3.35 \\
3.35 \\
2.62 \\
2.36 \\
2.33 \\
1.92 \\
1.45 \\
1.39 \\
1.38 \\
1.23 \\
1.09\end{array}$ & $\begin{array}{l}\text { Bronchitis } \\
\text { Intracranial angioma } \\
\text { Behavior disorder } \\
\text { Epilepsy } \\
\text { Nephritis } \\
\text { Nephritis } \\
\text { Cirrhosis } \\
\text { Nephrosis } \\
\text { Diabetes } \\
\text { Intestinal parasites } \\
\text { Osteodystrophy } \\
\text { Erythroblastic anemia } \\
\text { Cirrhosis } \\
\text { Cirrhosis } \\
\text { Sickle cell anemia } \\
\text { Cirrhosis } \\
\text { Erythroblastic anemia } \\
\text { Hemolytic anemia (un- } \\
\text { classified) }\end{array}$ \\
\hline
\end{tabular}

not greatly impaired. Patients 8, 9, and 10, with constants from 3.35 to $2.62 \times 10^{-3} \mathrm{mgm}$. units, presented no other finding to suggest hepatic defect. Patient 11 exhibited the peculiar syndrome of asymmetrical osteodystrophy with areas of pigmented skin which has been described by McCune (15) and by Albright (16). She herself showed no sign definitely attributable to liver malfunction; however, other patients with the same syndrome $(15,17)$ have given a story of severe and protracted neonatal icterus. The last seven cases in the table represent instances either of chronic hemolytic anemia and hyperbilirubinemia or of cirrhosis. In all of these there is reason to think that excretory hepatic function was impaired.

Review of all of the findings indicates first that excretory liver function exhibits in different subjects variations through a fairly wide range before it can be designated as impaired. It follows that some patients may as the result of disease suffer considerable loss of this power and still retain more adequate function than other normal subjects. The range of excretory power in individuals with functionally adequate livers aids in an understanding of the wide range of serum bilirubin levels encountered in normal subjects. In our experience these levels have varied from 0.21 to $0.99 \mathrm{mgm}$. per $100 \mathrm{cc}$. The findings also suggest that values for the excretory constant below $2.0 \times 10^{-3} \mathrm{mgm}$. units must be regarded as evidence of impaired function. With values between 2.0 and $2.5 \times 10^{-3} \mathrm{mgm}$. units, it is probable that function has been damaged. With constants above $2.5 \times 10^{-3} \mathrm{mgm}$. units, the significance of individual findings is less clear. If all of the constants above $2.5 \times 10^{-3} \mathrm{mgm}$. units, with the exception of the finding in the cirrhotic patient ( $R$. $\mathrm{H}$.), are accepted as apt to be encountered in the absence of hepatic defect, there are 26 records to determine the range and distribution of normal values. In mgm. units $\times 10^{-3}$ these records disclose : 2.5 to $4.0,5$ cases; 4.0 to $5.5,8$ cases ; 5.5 to $7.0,5$ cases ; 7.0 to $8.5,4$ cases; 8.5 to $10.0,1$ case; above 10.0, 3 cases.

\section{SUMMARY}

The rate of removal from the circulation of intravenously injected bilirubin can be evaluated as a "velocity constant of excretion" which provides a measure of the excretory function of the liver. The measure is not affected, as is the older type of bilirubin excretion test, by elevation of the basal plasma concentration.

Evaluation of the velocity constant is based on the assumption that normally circulating bilirubin depends on an equilibrium between the rate of formation, which is constant, and the rate of excretion, which has been observed to be approximately proportional at any moment to the square of the concentration in the plasma.

The velocity constant is computed in milligram units which express the rate of excretion from each 100 cc. of plasma in milligrams per minute per milligram-squared of concentration in the circulating plasma. A minimum of three determinations of serum bilirubin concentration is required for the calculation. The level before injecting bilirubin, $L_{\text {eq. }}$, is measured first. The injection, 5 mgm. per kilogram of body weight, is then given. After a lapse of 5 to 7 minutes for mixing in the circulation, a second sample, $L_{1}$, is obtained. Finally, when from 2 to 4 hours have elapsed, the third sample, $L_{2}$, is withdrawn. If $t_{1}$ and $t_{2}$ represent the time in minutes corresponding to the samples $L_{1}$ and $L_{2}$, which express concentration in milligrams per $100 \mathrm{cc}$. serum, the value of the velocity constant, $K$, can be computed from the equation :

$$
K=\frac{1.1513}{L_{\text {eq. }}\left(t_{2}-t_{1}\right)} \log \frac{\left(L_{2}+L_{\text {eq. }}\right)\left(L_{1}-L_{\text {eq. }}\right)}{\left(L_{2}-L_{\text {eq. }}\right)\left(L_{1}+L_{\text {eq. }}\right)} .
$$

A study of 35 patients with normal and defective livers revealed variations in the velocity constant from 1.09 to $24.5 \times 10^{-3} \mathrm{mgm}$. units. 
For the present, values above $2.5 \times 10^{-3} \mathrm{mgm}$. units must be interpreted as evidence of normal hepatic function, although low constants within the normal range may for some patients be indicative of loss of excretory power. Values between 2.0 and $2.5 \times 10^{-3} \mathrm{mgm}$. units provide presumptive evidence of damaged hepatic function and values below $2.0 \times 10^{-3} \mathrm{mgm}$. units are positive evidence that function is impaired.

\section{APPENDIX-METHOD OF ANALYSIS}

The assessment of hepatic function by means of bilirubin clearance depends upon accuracy in determining bilirubin in serum or plasma. In common with other investigators $(1,3)$ we have found that the requisite precision is not obtainable through measurement of the color developed in the van den Bergh reaction. This is true even when the azobilirubin is estimated photoelectrically by the excellent method of Malloy and Evelyn (18). Accordingly, we have followed the suggestions of Ernst and Förster (19) and of Eilbott (1) in using acetone to precipitate serum protein so that the yellow color of bilirubin itself can be measured in the filtrate. The sensitivity of the method and the accuracy of the results have been increased significantly by use of a selective color filter and photoelectric analysis. Previous investigators who have used the method for measuring bilirubin excretion have not been concerned with the error due to the yellow color of lipochromes; such carotenoid pigments presumably do not vary throughout the test and their absolute magnitude was unimportant since only increments in bilirubin concentration above the basal level were used in computing percentage retention. Measurement of the velocity of bilirubin clearance, however, depends upon the absolute concentration of bilirubin at all times during the test. Accordingly, carotenoid pigments have been estimated separately after extracting the serum with petroleum ether; a correction is then applied in the measurement of bilirubin.

Estimation of total yellow color (bilirubin plus lipochrome). Two and five-tenths cc. of distilled water, 0.5 cc. of the serum, and $10 \mathrm{cc}$. of redistilled acetone are pipetted in the order mentioned into a $15 \mathrm{cc}$. centrifuge tube. The contents are mixed thoroughly with a stirring rod which is subsequently discarded so that the tube can be closed with a rubber cap. It is then placed in the icebox for 15 minutes or until well chilled. Throughout these and later manipulations every effort is made to minimize exposure to light. We have routinely stored samples and tubes in a covered can, removing them only in subdued light for essential steps in the procedure. Centrifugation of the chilled tubes is carried out for 15 minutes in $300 \mathrm{cc}$. bronze cups which are filled with cracked ice before the tubes are inserted. Immediately afterwards the supernatant fluid is transferred to another tube which is stoppered and allowed to come to room temperature. The purpose of chilling during this phase of the procedure is to obtain a solution free from any trace of turbidity. When chilling is neglected the fluid will become warm while in the centrifuge and, even though clear when first removed, may later develop a slight cloudiness as the temperature falls. The final step involves the photoelectric reading.

Estimation of the yellow of lipochrome. Since it is assumed that the amount of carotenoid pigment in the serum does not vary during the test, the portions of the serial samples which remain after the estimation of total yellow color are mixed to provide a single sample on which lipochrome is measured. In principle the analysis follows the procedure of Stoner (20). Two cc. of the serum is transferred to a test tube; anhydrous calcium sulphate is added and by means of a glass spatula thoroughly mixed with the serum until a dry paste results. Two cc. of 95 per cent alcohol is stirred thoroughly with the paste. Then, exactly $8 \mathrm{cc}$. of petroleum ether is added; the tube is stoppered and the contents shaken vigorously for several minutes. Upon settling, a portion of the petroleum ether layer, which now contains the carotenoid pigments, is removed for measurement of color intensity in the photoelectric instrument.

With both analyses, total yellow color and lipochrome, the galvanometer readings are calculated to express in milligrams per $100 \mathrm{cc}$. of serum the quantity of bilirubin which would have yielded the same readings. Bilirubin is then computed as total yellow color minus the yellow due to lipochrome. With most subjects the correction for lipochrome is small, in the neighborhood of 0.1 or 0.2 $\mathrm{mgm}$. per $100 \mathrm{cc}$. In one patient only in our series, a diabetic, the correction reached a value of $0.67 \mathrm{mgm}$. per $100 \mathrm{cc}$.

The photoelectric photometer used for the estimation of color intensity was one which was constructed in our own laboratory (21); other instruments which utilize selective color filters should be adaptable to the procedure. Readings were made in optical cells which were either 2.5 or $5.0 \mathrm{~cm}$. in length; the shorter cell was used when color intensity was high and the longer cell with paler solutions. The filter, which furnishes blue light, was constructed of the following components: Corning Number 511, violet glass, thickness $3 \mathrm{~mm}$.; Jena optical filter glass GG-5, thickness $2 \mathrm{~mm}$; Corning light shade Aklo glass Number 396, thickness $2 \mathrm{~mm}$. Solutions of bilirubin exhibit maximum light absorption at $440 \mathrm{~m} \mu$; the filter described transmits light from 410 to $490 \mathrm{~m} \mu$, with a peak transmission at $450 \mathrm{~m} \mu$.

Calibration presented a problem. When different standard solutions are prepared by dissolving weighed amounts of bilirubin in chloroform and subsequently diluting either with alcohol or acetone, discrepancies among replicate readings will be much greater than when multiple measurements are made on the same solution. This is especially so when the bilirubin of one manufacturer is compared with that of another. We have assumed, then, that part of the difficulty arises from impurities in available samples of bilirubin; another part undoubtedly comes from the circumstance that bilirubin in either alcohol or acetone is not in true solution but rather in a state of colloidal dispersion. Variation in the size of such colloidal aggregates is known to influence light absorption 
(22). On the other hand, calibration curves prepared from dilutions of solutions showing the same initial color intensity have always agreed closely; likewise, the agreement between duplicate analyses of serum has been excellent. Evidently, then, the method is capable of yielding results whose relative precision is high; the absolute accuracy of the levels is not better than can be obtained by other good technics. These circumstances determined the method of calibration. The relative value of readings on the galvanometer scale was first determined carefully. The absolute concentration of the solutions of bilirubin used for this purpose was not known. Carefully prepared dilutions of each solution revealed slight but clearly demonstrable deviation from Beer's law. The deviations were calibrated so that color intensity could be measured in arbitrary units of bilirubin. Finally, the number of milligrams corresponding to each arbitrary unit was determined in the following way: Twenty-four samples of serum which contained varying amounts of indirect bilirubin were analyzed by the procedure outlined above and by the photoelectric method of Malloy and Evelyn (18). The latter method had previously been calibrated in our own laboratory with pure Hoffmann$\mathrm{La}$ Roche bilirubin. The average findings by the two methods were then used to determine the milligrams to be assigned to each unit. An important circumstance in leading us to adopt this comparative method of calibration was the fact that Hoffmann-La Roche bilirubin can no longer be purchased on the market. Malloy and Evelyn have presented reasons for believing this bilirubin to be of a higher grade of purity than that procurable from other sources.

Practically all of our analyses of serum have been performed in duplicate. The relative accuracy of the present method is indicated by the agreement between the duplicates. With levels of bilirubin below $1.0 \mathrm{mgm}$. per 100 cc., the data reveal a standard error of analysis, S.E.A, of $\pm 0.012 \mathrm{mgm}$. per $100 \mathrm{cc}$. With higher concentrations, the errors per $100 \mathrm{cc}$. are as follows: 1.0 to $2.0 \mathrm{mgm}$., S.E.A $=0.023 \mathrm{mgm}$.; 2.0 to $5.0 \mathrm{mgm}$., S.E.A $=0.029 \mathrm{mgm}$.; above $5.0 \mathrm{mgm}$., S.E.A $=0.045 \mathrm{mgm}$. The values yielded by the method do not appear to be affected by hemolysis in the sample. We have obtained identical results in serum analyzed before and after the addition of laked red blood cells. In contrast, such additions have always decreased the intensity of color in the van den Bergh reaction. The method cannot be used to measure bilirubin in the serum of patients with obstructive jaundice or with icterus due to hepatitis. A part of the bilirubin in these cases gives a direct reaction in the van den Bergh test; direct bilirubin is precipitated by acetone along with the serum proteins. Under such circumstances the protein precipitate is stained yellow and the fact that the estimate will be unreliable can be recognized at once.

\section{BIBLIOGRAPHY}

1. Eilbott, W., Funktionsprüfung der Leber mittels Bilirubinbelastung. Ztschr. f. klin. Med., 1927, 106, 529.
2. Von Bergmann, G., Zur funktionellen Pathologie der Leber inbesondere der Alkohol-Ätiologie der Cirrhose. Klin. Wchnschr., 1927, 6, 776.

3. Harrop, G. A., and Barron, E. S. G., The excretion of intravenously injected bilirubin as a test of liver function. J. Clin. Invest., 1931, 9, 577.

4. Soffer, L. J., Bilirubin excretion as a test for liver function during normal pregnancy. Bull. Johns Hopkins Hosp., 1933, 52, 365.

5. Soffer, L. J., and Paulson, M., Residual hepatic damage in catarrhal jaundice as determined by the bilirubin excretion test. Arch. Int. Med., 1934, 53, 809.

6. Soffer, L. J., Present day status of liver function tests. Medicine, 1935, 14, 185.

7. Soffer, L. J., and Paulson, M., Comparative advantages and further modification of the bilirubin excretion test for hepatic function. Am. J. M. Sc., 1936, 192, 535.

8. Campbell, A. D., and Soffer, L. J., The results of the bilirubin test for liver function on patients recovered from arsphenamine jaundice. Am. J. Syph., Gonor. and Ven. Dis., 1937, 21, 420.

9. Jankelson, I. R., and Gargill, S. L., Bilirubin liver function test. I. Modification of method. New England J. Med., 1931, 204, 547.

10. Ruhbaum, W., and Matheja, W., Leberfunktionsproben bei latenter Leberschädigung. Klin. Wchnschr., 1935, 14, 1568.

11. Mills, M. A., and Dragstedt, C. A., Removal of intravenously injected bromsulphalein from the blood stream of the dog. Arch. Int. Med., 1938, 62, 216.

12. Lyon, R. A., The bilirubin liver function test in the toxemias of pregnancy. Am. J. Obst. and Gynec. 1938, 36, 99.

13. Lin, H., and Eastman, N. J., The behavior of intravenously injected bilirubin in newborn infants. Am. J. Obst. and Gynec., 1937, 33, 317.

14. Gilligan, D. R., and Blumgart, H. L., Personal communication.

15. McCune, D. J., and Bruch, H., Osteodystrophia fibrosa. Am. J. Dis. Child., 1937, 54, 806.

16. Albright, F., and others, Syndrome characterized by osteitis fibrosa disseminata, areas of pigmentation and endocrine dysfunction, with precocious puberty in females. New England J. Med., 1937 216, 727.

17. Braid, F., Osseous dystrophy following icterus gravis neonatorum. Arch. Dis. Child., 1939, 14, 181.

18. Malloy, H. T., and Evelyn, K. A., The determination of bilirubin with the photoelectric colorimeter. J. Biol. Chem., 1937, 119, 481.

19. Ernst, Z., and Förster, J., Uber die Bestimmung des Blutbilirubins. Klin. Wchnschr., 1924, 3, 2386.

20. Stoner, W. C., Carotinemia. Am. J. M. Sc., 1928, $175,31$.

21. Weech, A. A., A micro-photoelectric photometer. Proc. Soc. Exp. Biol. and Med., 1940, 45, 858.

22. Fischer, H., and Orth, H., Die Chemie des Pyrrols, II Band, 1 Hälfte, Akademische Verlagsgesellschaft, Leipzig, 1937. 